\title{
Multiplicative-Binomial Distribution: Some Results on Characterization, Inference and Random Data Generation
}

\author{
Elsayed A.H. Elamir \\ Department of Mathematics and Statistics, Faculty of Commerce, Benha University, Egypt \\ \& \\ Management and Marketing Department, College of Business, University of Bahrain, \\ P.O. Box 32038, Kingdom of Bahrain \\ shabib40@gmail.com
}

Received 20 October 2012

Accepted 15 February 2013

\begin{abstract}
Multiplicative-binomial distribution is one of the distributions that allows for over-dispersion, and underdispersion relative to the standard binomial distribution. It will be shown that the multiplicative-binomial distribution can be a very useful model for these situations. Moreover, the confidence interval for the parameters of the multiplicative-binomial distribution is investigated by the profile likelihood methods. The first four moments and simulation procedures for generating data from the multiplicative-binomial distribution using R-software are given. By using four applications to simulated and real data it is shown that the multiplicative-binomial distribution is the same as or outperforming the standard binomial and beta-binomial distributions.
\end{abstract}

Keywords: binomial distribution; likelihood ratio test; over-dispersion; profile likelihood; zero-inflated data.

\section{Introduction}

The standard binomial distribution provides a standard framework for the analysis of count data under the assumptions of independent trials and fixed trial probability. In recent years there has been considerable interest in models for count data that allows for over-dispersion, under-dispersion and excess zeros relative to the standard binomial distribution. Application areas are diverse; see, Lambert (1992), Bowman and George (1995), Kibria (2006), Banik and Kibria (2009), Kolev and Paiva (2008) Siegel and Castellan (1988) and Yu and Zelterman (2002, 2008).

The Lovison's multiplicative-binomial distribution (LMBD) is introduced by Lovison (1998) as a generalization of the standard binomial distribution in the case of dependence among the trials that allows for over-dispersion and under-dispersion and includes the standard binomial distribution as a special case. In this paper, it will be argued that the LMBD could be used to model data with over-dispersion and under-dispersion relative to the standard binomial distribution. Moreover, appropriateness of the standard binomial distribution and confidence intervals for the LMBD parameters are investigated by the likelihood ratio test and the profile likelihood method, respectively. The 
first four moments and simulation procedures for generating random variates from LMBD using Rsoftware are studied. Four applications to simulated and real data and comparisons with binomial and beta-binomial distributions are given.

In Section 2 the LMBD is reviewed and its moments are obtained. The Likelihood ratio test and profile likelihood for the LMBD are studied in Section 3. The procedures for generating random variates from LMBD are given in Section 4. Four applications to simulated and real data and comparison with binomial and beta-binomial distributions are presented in Section 5. Section 6 is devoted to the conclusion.

\section{Lovison's multiplicative-binomial distribution}

Consider the random vector $\mathbf{Z}=\left[Z_{1}, \ldots, Z_{n}\right], Z_{i}$ being a binary response which measures whether some event of interest is present, ' 1 ' or absent, ' 0 ' for a sample of $n$ units and let $Y_{n}=\sum_{i=1}^{n} Z_{i}$ denote the sample frequency of successes. To accommodate for the possible dependence among trials and under the assumption that the units are exchangeable, Lovison (1998) proposed an alternative form of the multiplicative-binomial distribution introduced by Altham (1978) based on original Cox's representation (1972) as

$$
P\left(Y_{n}=y\right)=\frac{\left(\begin{array}{l}
n \\
y
\end{array}\right) \psi^{y}(1-\psi)^{n-y} \omega^{y(n-y)}}{\sum_{t=0}^{n}\left(\begin{array}{l}
n \\
t
\end{array}\right) \psi^{t}(1-\psi)^{n-t} \omega^{t(n-t)}}, \quad y=0,1, \ldots, n
$$

where $0<\psi<1$ and $\omega>0$ are the parameters; for more details about this distribution; see, Lovison (1998). This distribution provides a wider range of distributions than is provided by the standard binomial distribution and includes the standard binomial distribution as a special case at $\omega=1$.

Figures 1 and 2 show the distribution of $Y_{n}$ for $n=10, \psi=0.25,0.95$, and different values of $\omega$. For small values of $\psi$ and $\omega<1$ the distribution gives higher probability for zero than the standard binomial distribution. Also, for large values of $\psi$ and $\omega>1$ the distribution gives higher probability for values at $n$.

\subsection{Moments of LMBD}

Let

$$
\begin{gathered}
n_{(i)}=n(n-1) \cdots(n-i+1), \quad n_{(0)}=1, \\
\pi_{i}=\psi^{i} \frac{\kappa_{n-i}(\psi, \omega)}{\kappa_{n}(\psi, \omega)},
\end{gathered}
$$

and

$$
\kappa_{n-a}(\psi, \omega)=\sum_{x=0}^{n-a}\left(\begin{array}{c}
n-a \\
x
\end{array}\right) \psi^{x}(1-\psi)^{(n-a-x)} \omega^{(n-a-x)(x+a)}
$$



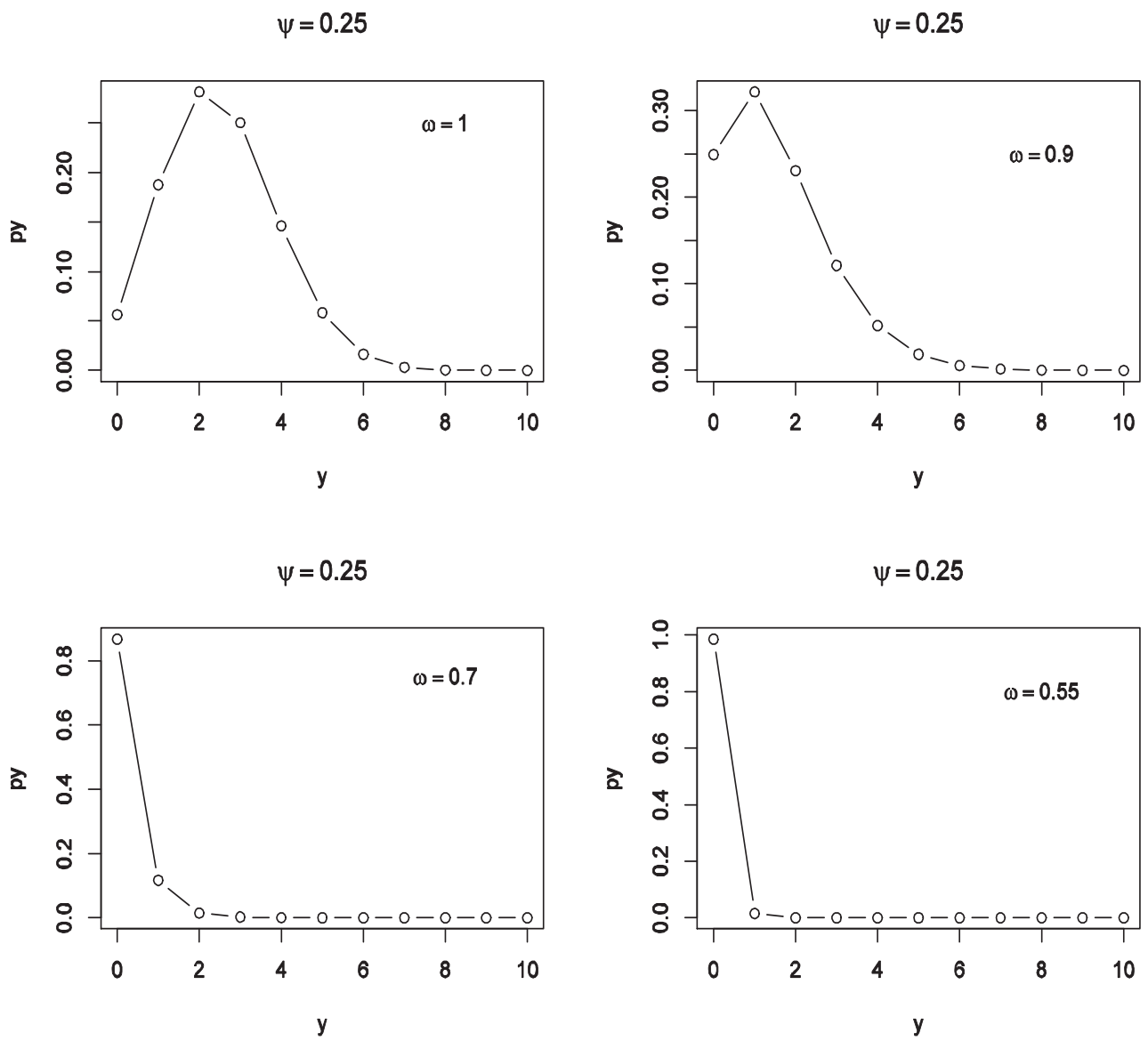

Fig. 1. LMB distribution for selected values of $\omega=1,0.9,0.7$, and $0.56 \psi=0.25$ and $n=10$.

The factorial moments for LMBD can be obtained as

$$
\mu_{(i)}=E(Y \cdots(Y-i+1))=\sum_{i=0}^{n} y \cdots(y-i+1) \frac{\left(\begin{array}{l}
n \\
y
\end{array}\right) \psi^{y}(1-\psi)^{n-y} \omega^{y(n-y)}}{\sum_{t=0}^{n}\left(\begin{array}{c}
n \\
t
\end{array}\right) \psi^{t}(1-\psi)^{n-t} \omega^{t(n-t)}}=n_{(i)} \pi_{i} .
$$

For example,

$$
\mu_{(2)}=E(Y(Y-1))=\sum_{i=0}^{n} y(y-1) \frac{n(n-1)\left(\begin{array}{c}
n-2 \\
y-2
\end{array}\right) \psi^{y}(1-\psi)^{n-y} \omega^{y(n-y)}}{y(y-1) \sum_{t=0}^{n}\left(\begin{array}{c}
n \\
t
\end{array}\right) \psi^{t}(1-\psi)^{n-t} \omega^{t(n-t)}}=n_{(2)} \pi_{2}
$$

The first four non-central moments are

$$
\mu_{1}=n_{(1)} \pi_{1}=E(Y)=\mu,
$$



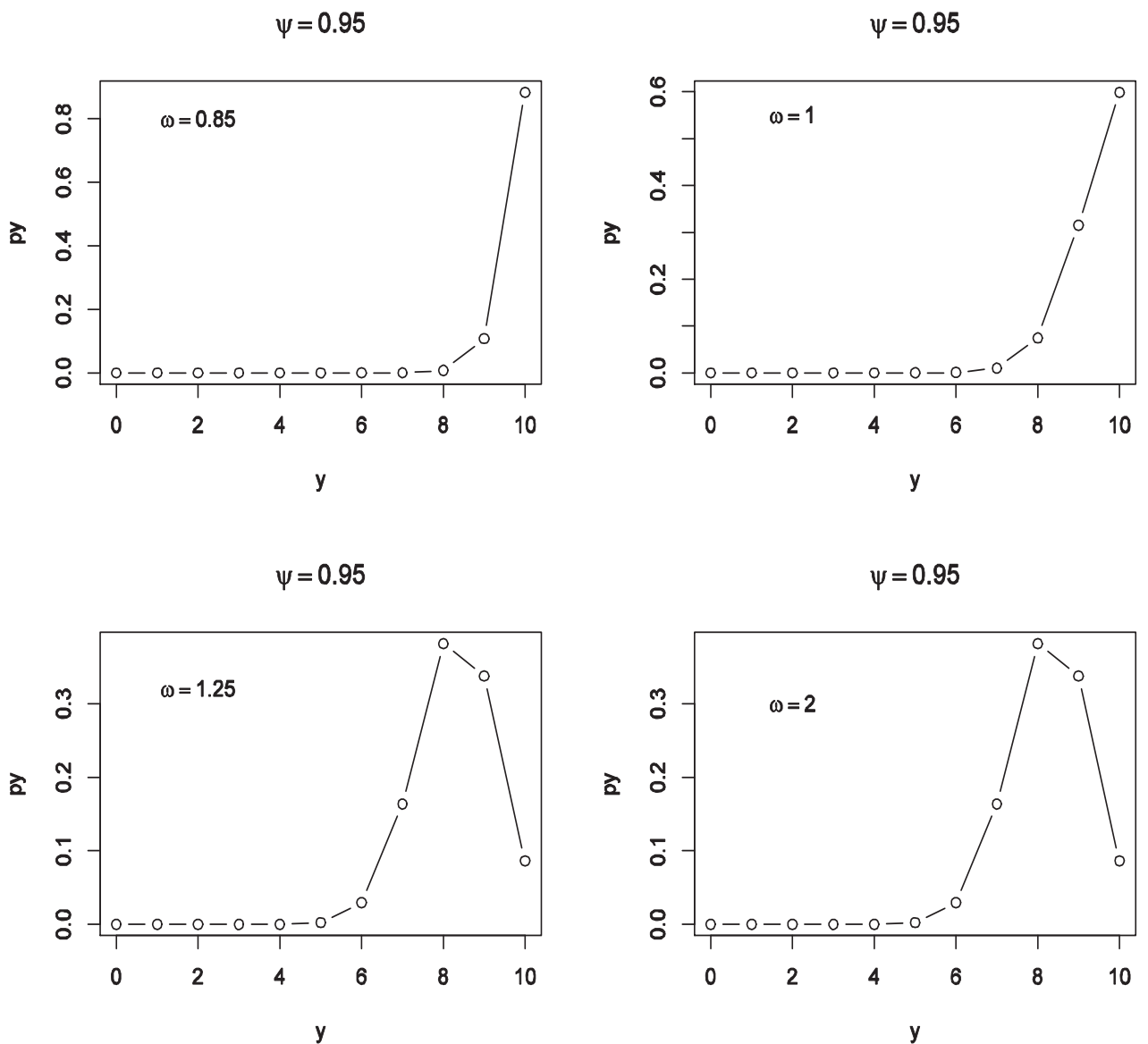

Fig. 2. LMB distribution for selected values of $\omega=0.85,1,1.25$, and 2 , and $\psi=0.95$ and $n=10$.

$$
\begin{gathered}
\dot{\mu}_{2}=n_{(1)} \pi_{1}+n_{(2)} \pi_{2}, \\
\dot{\mu}_{3}=n_{(1)} \pi_{1}+3 n_{(2)} \pi_{2}+n_{(3)} \pi_{3},
\end{gathered}
$$

and

$$
\mu_{4}=n_{(1)} \pi_{1}+7 n_{(2)} \pi_{2}+6 n_{(3)} \pi_{3}+n_{(4)} \pi_{4},
$$

The central moments are

$$
\begin{gathered}
\mu_{2}=n_{(1)} \pi_{1}+n_{(2)} \pi_{2}-n_{(1)}^{2} \pi_{1}^{2}=V(Y)=\sigma^{2}, \\
\mu_{3}=\left[n_{(1)} \pi_{1}+3 n_{(2)} \pi_{2}+n_{(3)} \pi_{3}\right]-3 n_{(1)} \pi_{1}\left[n_{(1)} \pi_{1}+n_{(2)} \pi_{2}\right]+2 n_{(1)}^{3} \pi_{1}^{3},
\end{gathered}
$$

and

$$
\begin{aligned}
\mu_{4}=\left[n_{(1)} \pi_{1}+7 n_{(2)} \pi_{2}+6 n_{(3)} \pi_{3}+n_{(4)} \pi_{4}\right] & -4 n_{(1)} \pi_{1}\left[n_{(1)} \pi_{1}+3 n_{(2)} \pi_{2}+n_{(3)} \pi_{3}\right] \\
& +6 n_{(1)}^{2} \pi_{1}^{2}\left[n_{(1)} \pi_{1}+n_{(2)} \pi_{2}\right]-3 n_{(1)}^{4} \pi_{1}^{4}
\end{aligned}
$$




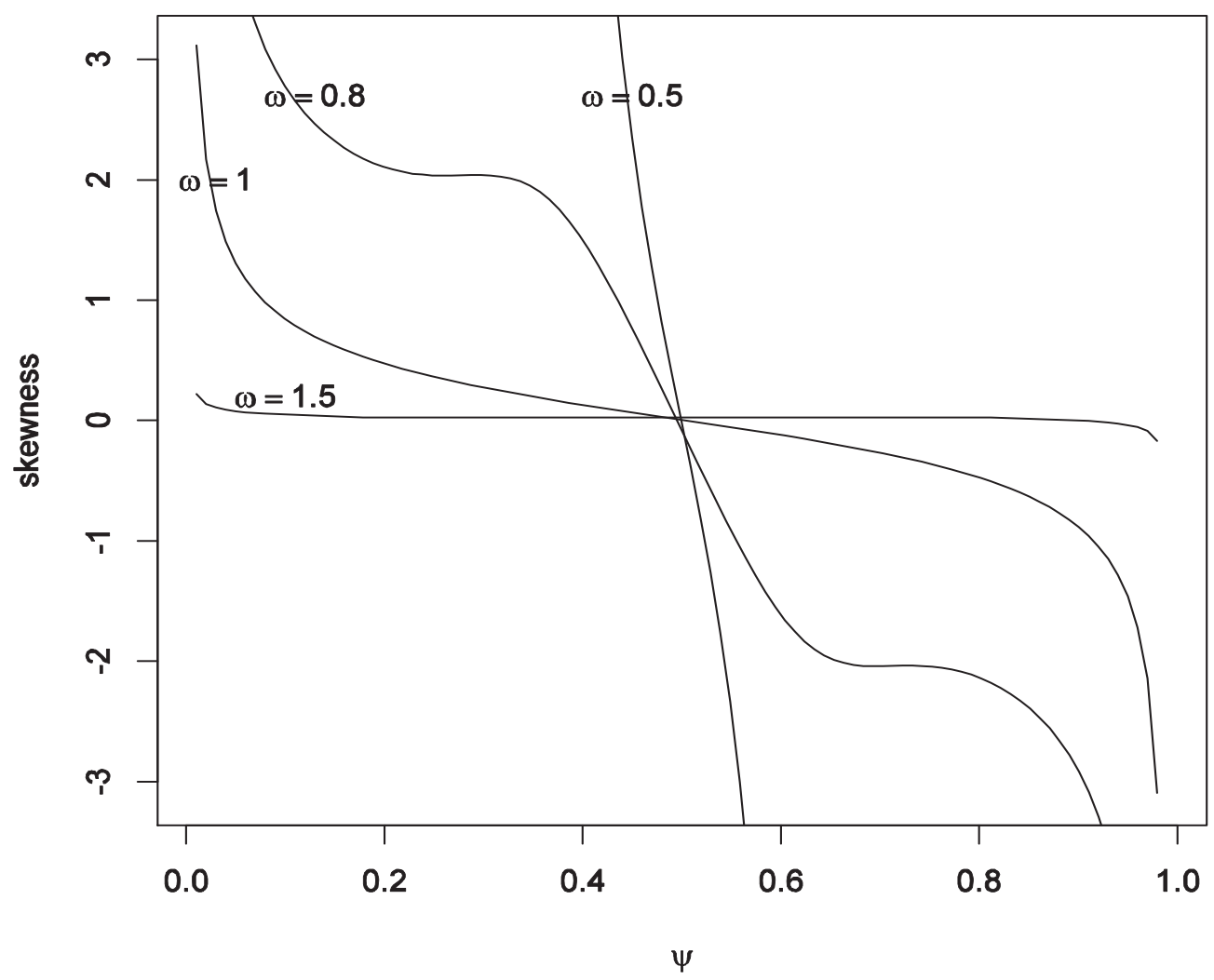

Fig. 3. Skewness $\gamma$ of LMBD with selected values of $\omega=0.5,0.8,1$, and 1.5 over the range $\psi=(0,1)$ and $n=10$.

The skewness measure is

$$
\gamma=\frac{\mu_{3}}{\left(\sigma^{2}\right)^{3 / 2}}
$$

The kurtosis measure is

$$
\beta=\frac{\mu_{4}}{\mu_{2}^{2}}
$$

Figures 3 and 4 show the skewness and kurtosis measures for some selected values of $\psi$ and $\omega$ at $n=10$.

From the expected value the relationships among, $p, \psi$, and $\omega$ are

$$
p=P(Z=1)=\psi \frac{\kappa_{n-1}(\psi, \omega)}{\kappa_{n}(\psi, \omega)}, \text { and } \psi=p \frac{\kappa_{n}(\psi, \omega)}{\kappa_{n-1}(\psi, \omega)} .
$$

Thus $p$ can be considered as a probability of success and $\psi$ as a probability of success weighted by the intra-units association measure $\omega$ governing the dependence among the binary responses of 


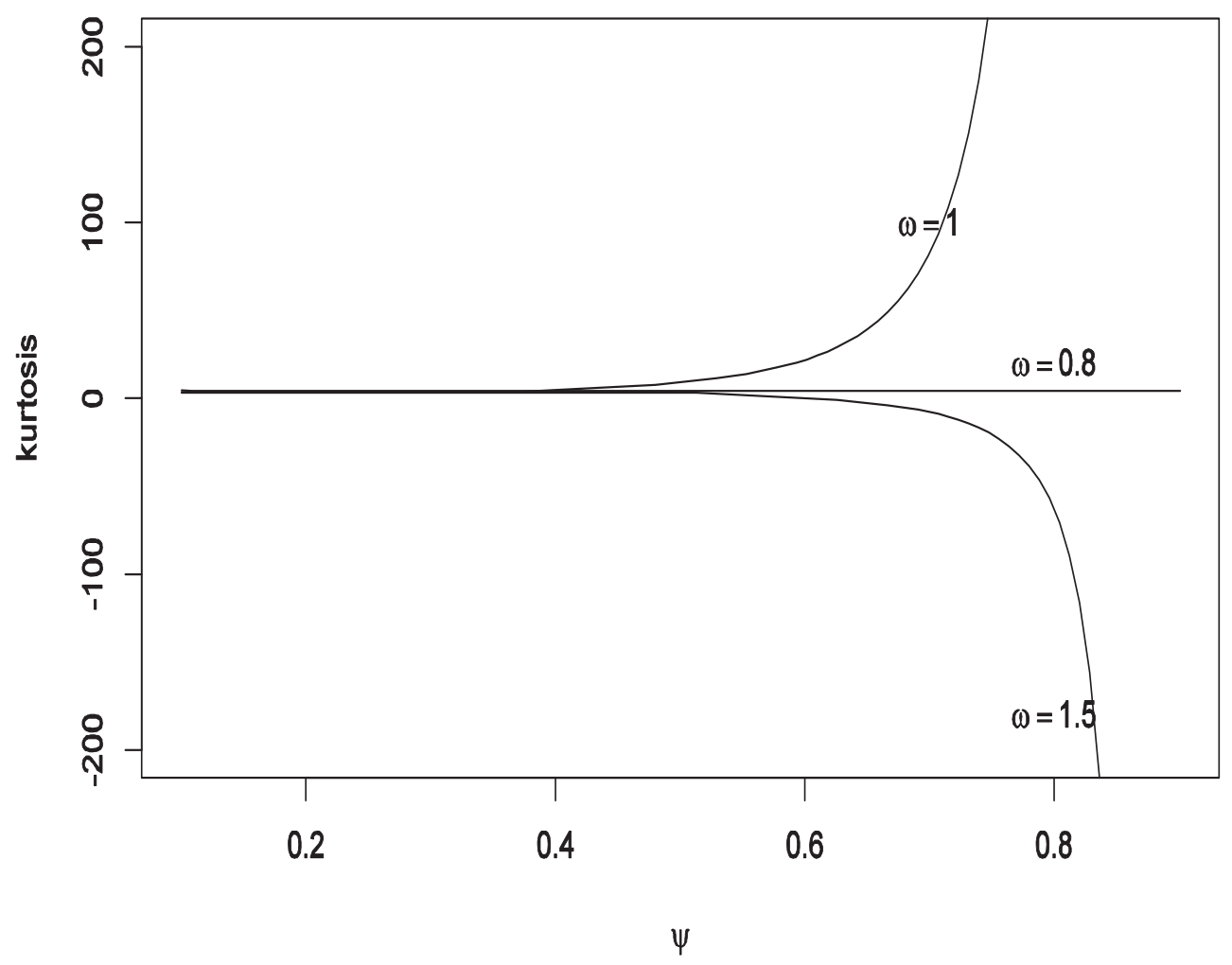

Fig. 4. Kurtosis $\beta$ of LMBD with selected values of $\omega=1,0.8$, and 1.5 over the range $\psi=(0,1)$ and $n=10$.

the $n$ units. If $\omega=1$ then $p=\psi$ represents the probability of success of the $n$ units when they are independent. If $\omega \neq 1$ the binary units are not independent and therefore $p \neq \psi$.

\section{Likelihood inference on the parameters of the LMBD}

When the LMBD fits the data, the profile likelihood and the likelihood ratio can be used to test the appropriateness of the standard binomial distribution and the profile likelihood can be used for estimating the confidence intervals for the LMBD parameters.

\subsection{Confidence intervals based on the profile likelihood}

When the analyst is interested in one of the parameters, the profile likelihood is a useful general tool obtained by maximizing out the remaining parameters for each fixed value of the parameter of interest. It provides a graphical summary of the most plausible values of the parameters of interest and can provide confidence intervals that are more accurate than the standard intervals based on normal theory; see, Pawitan (2001), Severini (2000) and Venzon and Moolgavkar (1988). From 
Habib (2010) the logarithm of the likelihood of the multiplicative-binomial can be written as

$$
l(\psi, \omega)=\log R !+\sum_{y=0}^{n} f_{y} \log P(y ; \psi, \omega)-\sum_{y=0}^{n} \log f_{y} !
$$

Note that $R$ is the number of sets of $n$ trials, $f_{y}$ the number of sets giving $y$ success, and $P(y ; \psi, \omega)$ is the LMBD. Given the joint likelihood $L(\psi, \omega)$ the profile likelihood of $\omega$ can be written as

$$
l_{p}(\omega)=\max _{\psi} l(\psi, \omega)
$$

where maximization of $\psi$ is performed at a fixed value of $\omega$. To obtain the confidence interval for $\omega$, the profile likelihood is assumed to be an increasing function to the left of its maximum and the following steps are used:

1. Compute the ML estimates $(\widehat{\psi}, \widehat{\omega})$ and the corresponding log-likelihood value,

2. Compute a "reasonable" lower and upper bound $\omega_{1}$ and $\omega_{2}$, respectively (e.g., $\widehat{\omega}-5 S E(\widehat{\omega})$ and) $\widehat{\omega}+5 S E(\widehat{\omega})$,

3. Define a grid of values ranging from $\omega_{1}$ to $\omega_{2}$.

4. For each grid value $\omega_{i}$ compute the profile $\log$-likelihood value $l_{p}\left(\omega_{i}\right)$ by maximizing the $l\left(\psi, \omega_{i}\right)$ over $\psi$-values,

5. Consider the smallest value of $\omega_{i}$ as the lower bound $\left(\omega_{L}\right)$ for the $(1-\alpha) \%$ CI such that $l_{p}\left(\omega_{i}\right) \geqslant$ $l_{p}(\widehat{\omega})-\frac{\chi_{1,1-\alpha}^{2}}{2}=l(\widehat{\psi}, \widehat{\omega})-\frac{\chi_{1,1-\alpha}^{2}}{2}$.

6. Consider the largest value of $\omega_{i}$ as the upper bound $\left(\omega_{U}\right)$ for the $(1-\alpha) \%$ CI such that $l_{p}\left(\omega_{i}\right) \geqslant$ $l_{p}(\widehat{\omega})-\frac{\chi_{1,1-\alpha}^{2}}{2}=l(\widehat{\psi}, \widehat{\omega})-\frac{\chi_{1,1-\alpha}^{2}}{2}$.

If necessary, refine or extend the grid of values around $\omega_{L}$ and $\omega_{U}$ to obtain greater accuracy.

Similarly in which $\psi$ is considered as the parameter of interest and $\omega$ is the nuisance parameter, the profile likelihood of $\psi$ can be written as

$$
l_{p}(\psi)=\max _{\omega} l(\psi, \omega)
$$

To obtain the confidence interval for $\psi$ the following steps can be used:

1. Compute the ML estimates $(\widehat{\psi}, \widehat{\omega})$ and the corresponding log-likelihood value,

2. Compute a "reasonable" lower and upper bound $\psi_{1}$ and $\psi_{2}$ respectively, (e.g., $\widehat{\psi}-5 S E(\widehat{\psi})$ and) $\widehat{\psi}+5 \operatorname{SE}(\widehat{\psi})$,

3. Define a grid of values ranging from $\psi_{1}$ to $\psi_{2}$.

4. For each grid value $\psi_{i}$ compute the profile $\log$-likelihood value $l_{p}\left(\psi_{i}\right)$ by maximizing the $l\left(\psi_{i}, \omega\right)$ over $\omega$-values,

5. Consider the smallest value of $\psi_{i}$ as the lower bound $\left(\psi_{L}\right)$ for the $(1-\alpha) \% \mathrm{CI}$ such that $l_{p}\left(\psi_{i}\right) \geqslant$ $l_{p}(\widehat{\psi})-\frac{\chi_{1,1-\alpha}^{2}}{2}=l(\widehat{\psi}, \widehat{\omega})-\frac{\chi_{1,1-\alpha}^{2}}{2}$,

6. Consider the largest value of $\psi_{i}$ as the upper bound $\left(\psi_{U}\right)$ for the $(1-\alpha) \%$ CI such that $l_{p}\left(\psi_{L}\right) \geqslant$ $l_{p}(\widehat{\psi})-\frac{\chi_{1,1-\alpha}^{2}}{2}=l(\widehat{\psi}, \widehat{\omega})-\frac{\chi_{1,1-\alpha}^{2}}{2}$.

If necessary, refine or extend the grid of values around $\psi_{L}$ and $\psi_{U}$ to obtain greater accuracy. 


\subsection{Likelihood ratio test for appropriateness of the standard binomial versus LMBD}

Consider a LMB distribution with likelihood function $L(\psi, \omega)$ and $(\widehat{\psi}, \widehat{\omega})$ the maximum likelihood (ML) estimates. The likelihood ratio test statistic $(\Lambda)$ of the hypothesis $H_{0}: \omega=1$ against $H_{1}: \omega \neq 1$ is

$$
\Lambda=2[\ln L(\widehat{\psi}, \widehat{\omega})-\ln L(\ddot{\psi}, 1)]=2[l(\widehat{\psi}, \widehat{\omega})-l(\ddot{\psi}, 1)]
$$

$\ddot{\psi}$ is the ML estimate of the reduced model. From (3.1) the model under the alternative hypothesis is

$$
l(\widehat{\boldsymbol{\psi}}, \widehat{\boldsymbol{\omega}})=\log R !+\sum_{y=0}^{n} f_{y} \log P(y ; \widehat{\boldsymbol{\psi}}, \widehat{\boldsymbol{\omega}})-\sum_{y=0}^{n} \log f_{y} ! .
$$

The model under the null hypothesis is

$$
l(\ddot{\psi}, 1)=\log R !+\sum_{y=0}^{n} f_{y} \log P(y ; \ddot{\psi}, 1)-\sum_{y=0}^{n} \log f_{y} ! .
$$

Hence,

$$
l(\widehat{\psi}, \widehat{\omega})-l(\ddot{\psi}, 1)=\sum_{y=0}^{n} f_{y} \log P(y ; \widehat{\psi}, \widehat{\omega})-\sum_{y=0}^{n} f_{y} \log P(y ; \ddot{\psi}, 1) .
$$

Therefore,

$$
\Lambda=2 \sum_{y=0}^{n} f_{y}[\log P(y ; \widehat{\psi}, \widehat{\omega})-\log P(y ; \ddot{\psi}, 1)]
$$

Under the null hypothesis, the test statistic $\Lambda$ approximately follows a chi-square distribution with one degree of freedom; see, Severini (2000).

\section{Generating random data from $L M B D$ using $R$}

The probability mass function for the LMB distribution is

$$
P(y ; \psi, \omega)=\frac{\left(\begin{array}{l}
n \\
y
\end{array}\right) \psi^{y}(1-\psi)^{n-y} \omega^{y(n-y)}}{\sum_{t=0}^{n}\left(\begin{array}{l}
n \\
t
\end{array}\right) \psi^{t}(1-\psi)^{n-t} \omega^{t(n-t)}}, \quad y=0, \ldots, n .
$$

Generating random data from this distribution for given $n, \psi$, and $\omega$ can easily be accomplished in $\mathrm{R}$ by using the probability mass function and the sample command, which has the following general syntax.

$>\backslash \# \mathrm{x}$ : a vector of elements from which to choose, size: \\# of item to choose $>$ sample $(x$, size, replace $=$ FALSE, prob $=$ NULL $)$

Example 4.1. This example is to draw 10000 and 100000 sample data from LMB distribution with $n=4, \psi=0.20$, and $\omega=0.45$. From the probability mass function of LMBD we obtain the probability distribution in Table 1.

The following command is used. 
Table 1. Exact and simulated mean and variance from LMBD with $n=4, \psi=0.20$, and $\omega=0.45$.

\begin{tabular}{cccccc}
\hline$y$ & 0 & 1 & 2 & 3 & 4 \\
$P(y)$ & 0.89597 & 0.08164 & 0.01377 & 0.00510 & 0.00349 \\
\multicolumn{5}{c}{ Simulated Size } \\
Mean & Exact & $\mathbf{0 . 1 3 8 5 1}$ & 0.13780 & 100000 & 0.13977 \\
Variance & $\mathbf{0 . 2 1 9 4 9}$ & 0.22303 & 0.21941 & & \\
Skewness & $\mathbf{4 . 4 7 1 0 1}$ & 4.50310 & 4.47905 & \\
\hline
\end{tabular}

$>\mathrm{x}=$ sample $(\mathrm{c}(0,1,2,3,4)$, size, replace=TRUE, prob= $c(0.89597,0.08164,0.01378,0.00510,0.00350))$

The size can be replaced by the desired value of 10000 and 100000 and therefore the variable $x$ contains random data drawn from the LMBD with $n=4, \psi=0.20$, and $\omega=0.45$. The mean, the variance and skewness of simulated data are compared with exact distribution in Table 1. A program in R-software to generate random data from LMBD (lmbd.sim) is given as follows:

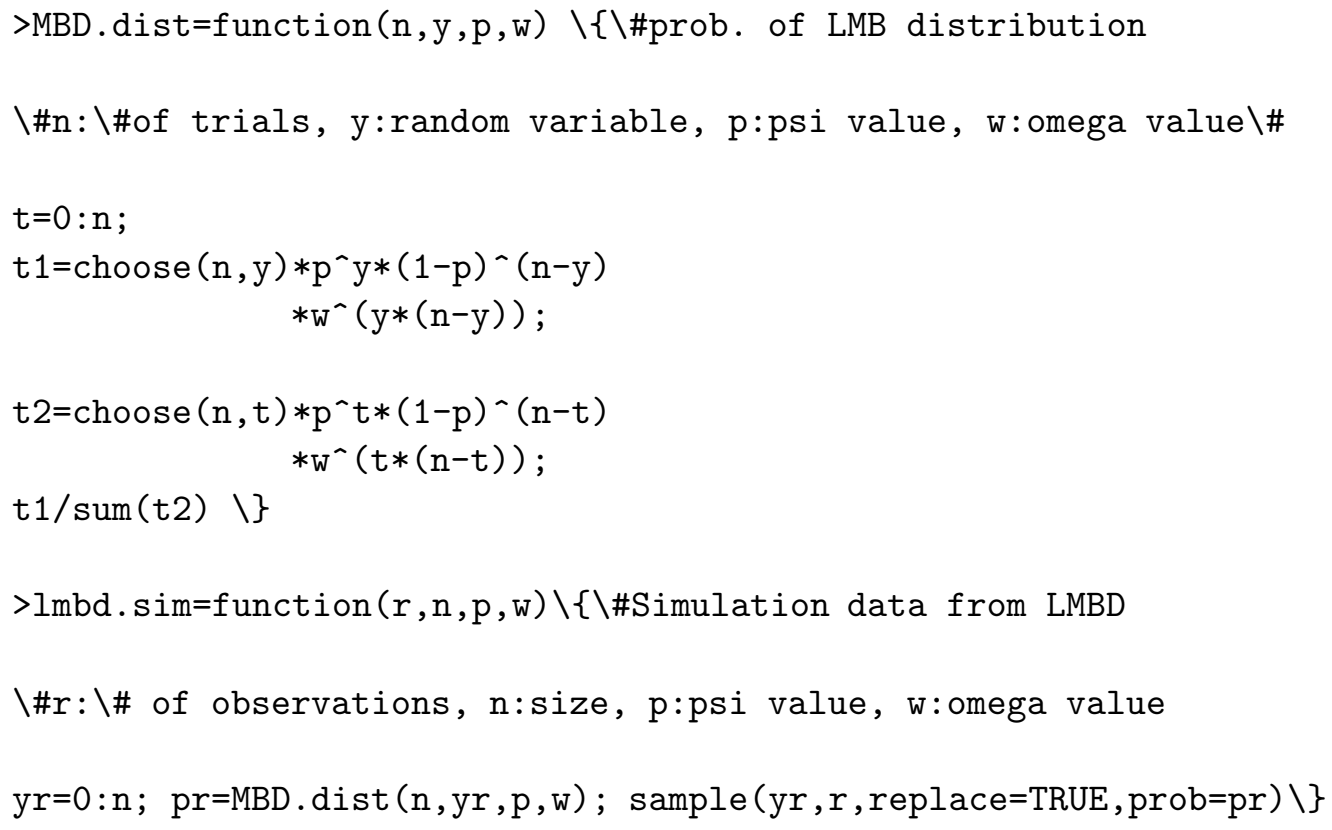

\section{Applications to simulated and real data}

Four applications to simulated and real data are studied. The first application deals with data simulated from a standard binomial. The second application presents data coming from a distribution which has variance less than the standard binomial. The third and fourth applications deal with real data with excess-zeros, which usually have more variance than the standard binomial. Moreover the useful and common-used beta-binomial distribution that is defined as

$$
f(x ; \alpha, \beta)=\left(\begin{array}{l}
n \\
x
\end{array}\right) \frac{B(\alpha+x, \beta+n-x)}{B(\alpha, \beta)}, \quad x=0,1, \ldots, n,
$$

is compared with LMBD in both simulated and real data analysis. The package "VGAM" in Rsoftware is used to estimate the parameters $\alpha$ and $\beta$. 
Table 2. Simulated data from binomial distribution with $n=4$ and $p=0.15$.

\begin{tabular}{lcccccc}
\hline \multicolumn{1}{c}{$y$} & 0 & 1 & 2 & 3 & 4 & Total \\
Obs. & 255 & 186 & 52 & 6 & 1 & 500 \\
E. LMBD & 256.46 & 183.67 & 52.42 & $\{7.07$ & $0.38\}$ & $X^{2}=0.068$ \\
E. binomial & 253.71 & 187.58 & 52.01 & $\{6.41$ & $0.30\}$ & $X^{2}=0.032$ \\
E. Beta-binomial & 254.87 & 185.75 & 52.30 & $\{6.74$ & $0.33\}$ & $X^{2}=0.003$ \\
\hline
\end{tabular}

Obs.: observed and E.: Expected value.

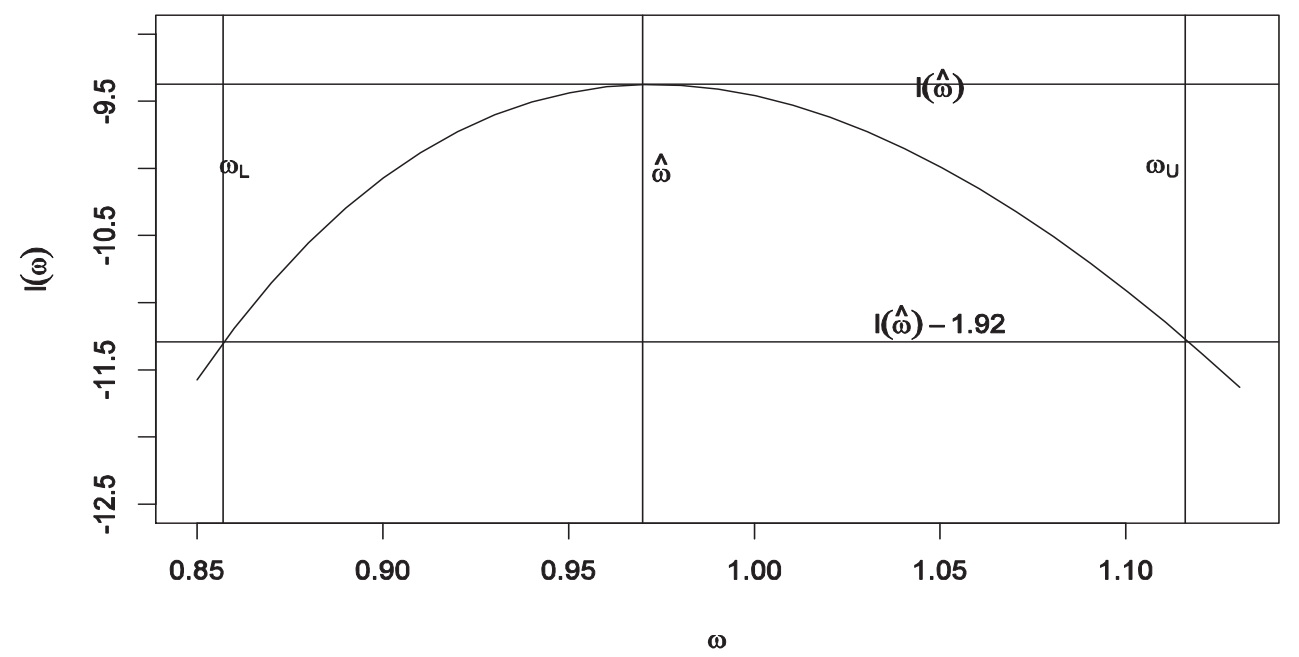

Fig. 5. Profile likelihood and 95\% CI: $\left(\omega_{L}, \omega_{U}\right)$ for the data in Application 1.

\subsection{Application 1: Simulated data from binomial}

The data are simulated from a standard binomial distribution with $n=4$ and $p=0.15$. The expected values and Pearson's $X^{2}$ statistic are given in Table 2. From Habib (2010) the maximum likelihood estimates for LMBD parameters are $\widehat{\psi}=0.164$ and $\widehat{\omega}=0.97$. The values of $X^{2}$ statistic in Table 2 show a suitable fit for the data by all the distributions.

Figure 5 shows the profile log-likelihood function for $\omega$ with its maximal value of -9.373 , and the $95 \%$ confidence interval $(0.857,1.116)$. Clearly, this C.I. suggests that a standard binomial distribution would be an appropriate model for the data, since $\omega=1$ is included in the interval. This can also be seen by the likelihood ratio test, $\Lambda=2(0.0827)=0.1654$ indicating the appropriateness of the standard binomial model.

\subsection{Application 2: Simulated data from LMBD}

The data are simulated from a LMBD with $n=6, \psi=0.5, \omega=1.25$, and variance 0.937 . The expected values and $X^{2}$ statistics are given in Table 3. From Habib (2010) the maximum likelihood estimates for LMBD parameters are $\widehat{\psi}=0.494$ and $\widehat{\omega}=1.22$. The estimate for $\widehat{p}$ is 0.496 . The values of $X^{2}$ statistics in Table 3 show a suitable fit for the data by the LMBD but not by the 
Table 3. Simulated data from LMBD with $n=6, \psi=0.5$, and $\omega=1.25$.

\begin{tabular}{lcccccccc}
\hline \multicolumn{1}{c}{$y$} & 0 & 1 & 2 & 3 & 4 & 5 & 6 & Total \\
Obs. & 3 & 25 & 129 & 194 & 122 & 26 & 1 & 500 \\
E. LMBD & $\{1.81$ & $28.35\}$ & 125.05 & 198.27 & 119.2 & $\{25.76$ & $1.56\}$ & $X^{2}=0.44$ \\
E. Binomial & 8.19 & 48.39 & 119.05 & 156.22 & 115.30 & 45.39 & 7.44 & $X^{2}=38.8$ \\
E. Beta-binomial & Not defined & & & & & & \\
\hline
\end{tabular}

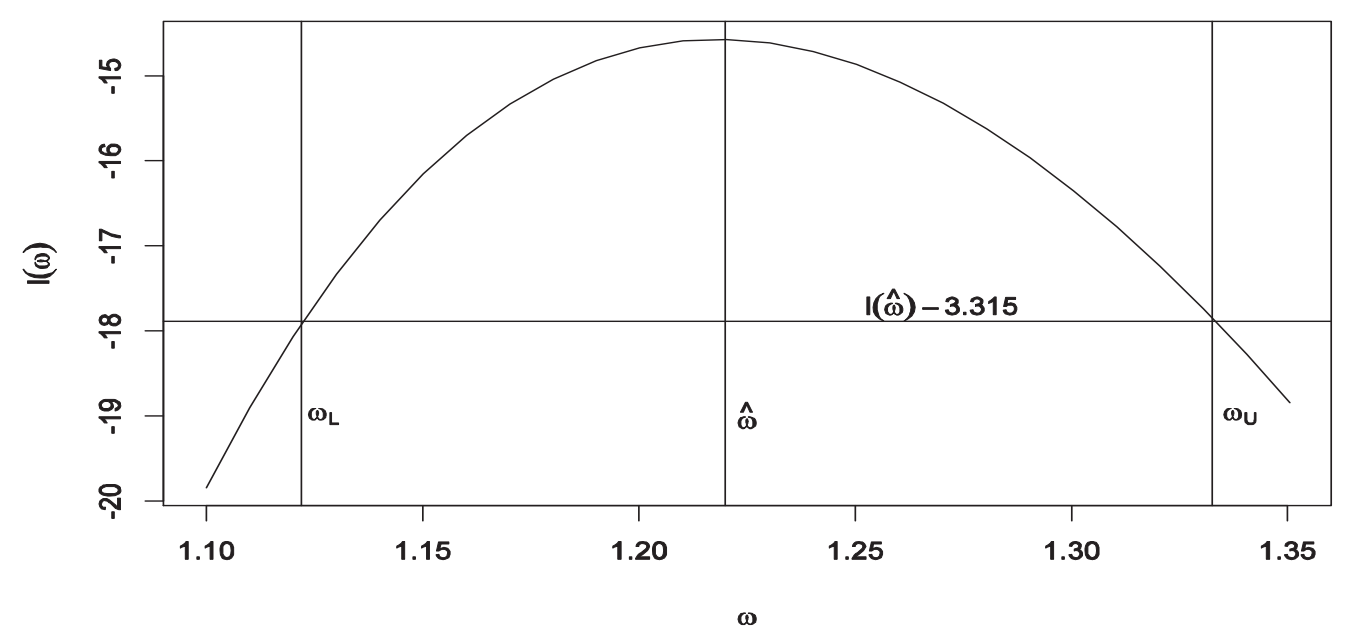

Fig. 6. Profile likelihood and 99\% CI: $\left(\omega_{L}, \omega_{U}\right)$ for the data in Application 2.

binomial model and the parameters of beta-binomial distribution cannot be estimated using package "VGAM".

Figure 6 shows the profile log-likelihood function for $\omega$ with its maximal value of -14.57 , and the $99 \%$ confidence interval $(1.122,1.332)$. Clearly, this C.I. suggests that a standard binomial distribution would not be an appropriate model for the data, since $\omega=1$ is not included in the interval. This can also be seen by the likelihood ratio test $\Lambda=2(22.44)=44.88$.

\subsection{Application 3: Traffic accident research}

Kuan et al. (1991) discuss data coming from the California Department of Motor Vehicles master driver license file. The variable of interest is the number of accidents per driver. A possible motivation can be seen in the possibility of finding risk factors involved in the accidents. The data, the expected values and $X^{2}$ are given in Table 4. These data have mean 0.203 and variance 0.235 and there is a clear spike of extra zeros (about 83\%) representing the accident-free drivers. From Habib (2010) the maximum likelihood estimates for LMBD parameters are $\widehat{\psi}=0.145$ and $\widehat{\omega}=0.58$. The estimate for $\widehat{p}$ is 0.067 . The values of $X^{2}$ statistics in Table 4 show a suitable fit for the data by the LMB and beta-binomial distributions but not by the binomial distribution.

Figure 7 shows the profile log-likelihood function for $\omega$ with its maximal value of -10.10 , and the $95 \%$ confidence interval $(0.539,0.622)$. Clearly, this C.I. suggests that a standard binomial 
Table 4. Distribution of the number of accidents per deriver.

\begin{tabular}{lccccc}
\hline number of accidents & 0 & 1 & 2 & 3 & Total \\
Number of drivers & 4499 & 766 & 136 & 21 & 5422 \\
Relative frequency & 0.830 & 0.141 & 0.025 & 0.004 & \\
E. LMB & 4499.38 & 770.07 & 130.60 & 21.95 & $X^{2}=0.286$ \\
E. binomial & 4403.56 & 948.68 & $\{68.12$ & $1.63\}$ & $X^{2}=334.9$ \\
E. Beta-binomial & 4497.7 & 764.65 & 142.81 & 16.82 & $X^{2}=1.36$ \\
\hline
\end{tabular}

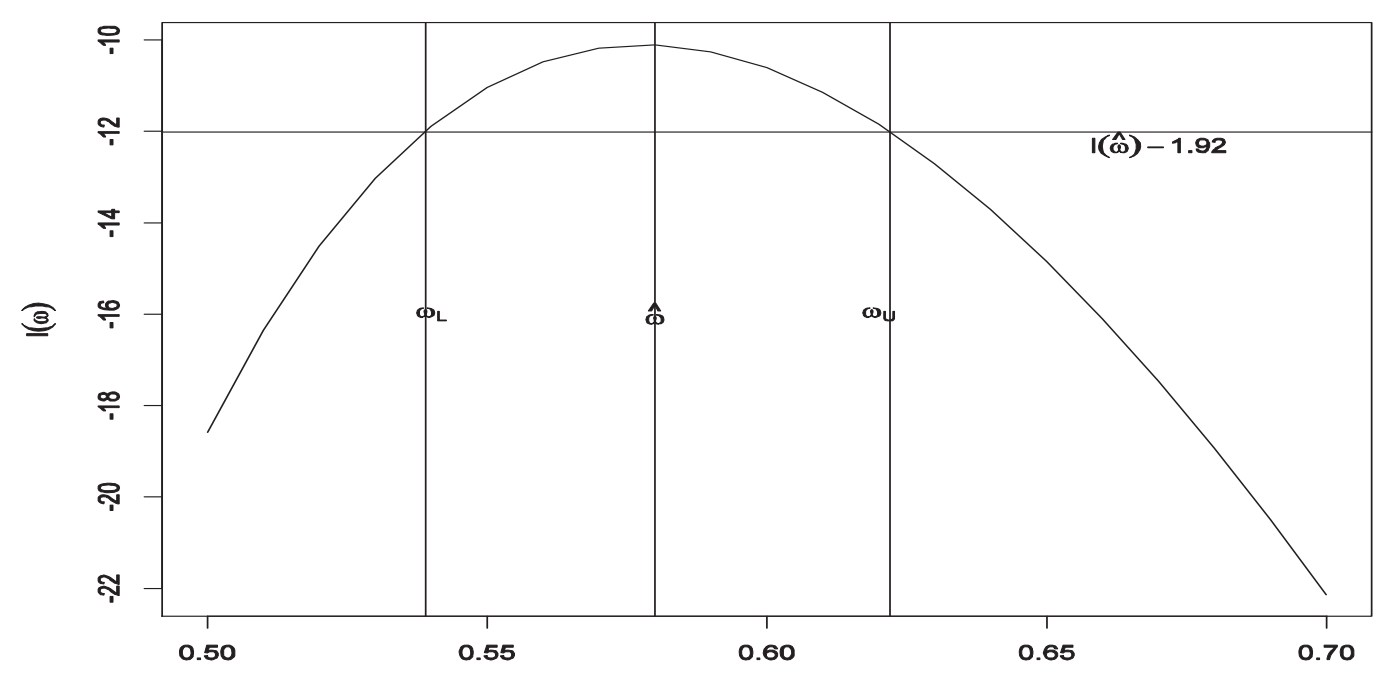

$\omega$

Fig. 7. Profile likelihood function and $95 \%$ CI: $\left(\omega_{L}, \omega_{U}\right)$ for the traffic accident research data.

distribution would not be an appropriate model for these data. This can also be seen by the likelihood ratio test $\Lambda=2(80.16)=160.33$.

\subsection{Application 4: Crime Sociology}

In a study on deviating behavior Bohning et al. (1997) provide an analysis of a data set on 4301 persons with criminal behavior. The variable of interest is the number of criminal acts per person. The motivation for such a study might be to find factors leading to deviating behavior. The data, the expected values and $X^{2}$ statistic are given in Table 5. These data have mean 0.0776 and variance 0.1209. There is a clear spike of extra zeros (about 94\%) representing acts-free criminals. From Habib (2010) the maximum likelihood estimates for LMBD parameters are $\widehat{\psi}=0.203$ and $\widehat{\omega}=$ 0.460 . The estimate for $\widehat{p}$ is 0.0155 . The values of $X^{2}$ statistics in Table 5 show a suitable fit for the data by the LMBD but not by the binomial model for all level of significance and the beta-binomial distribution at $10 \%$ and $5 \%$ level of significance.

Figure 8 shows the profile log-likelihood function for $\omega$ with its maximal value of -13.32 , and the $95 \%$ confidence interval $(0.435,0.485)$. Again, this C.I. suggests that a standard binomial 
Table 5. Distribution of the number of criminal acts.

\begin{tabular}{lccccccc}
\hline \# of criminal acts & 0 & 1 & 2 & 3 & 4 & 5 & Total \\
\# of persons & 4037 & 219 & 29 & 9 & 5 & 2 & 4301 \\
Relative frequency & 0.9386 & 0.0509 & 0.0067 & 0.0021 & 0.0012 & 0.0005 & \\
E. LMBD & 4032.66 & 229.38 & 24.66 & 6.27 & $\{3.76$ & $4.27\}$ & $X^{2}=2.55$ \\
E. Binomial & 3977.8 & 313.13 & $\{9.86$ & 0.155 & 0.0012 & $0\}$ & $X^{2}=151.3$ \\
E. Beta-binomial & 4030.66 & 218.40 & 42.14 & $\{8.34$ & 1.33 & $0.12\}$ & $X^{2}=8.1$ \\
\hline
\end{tabular}

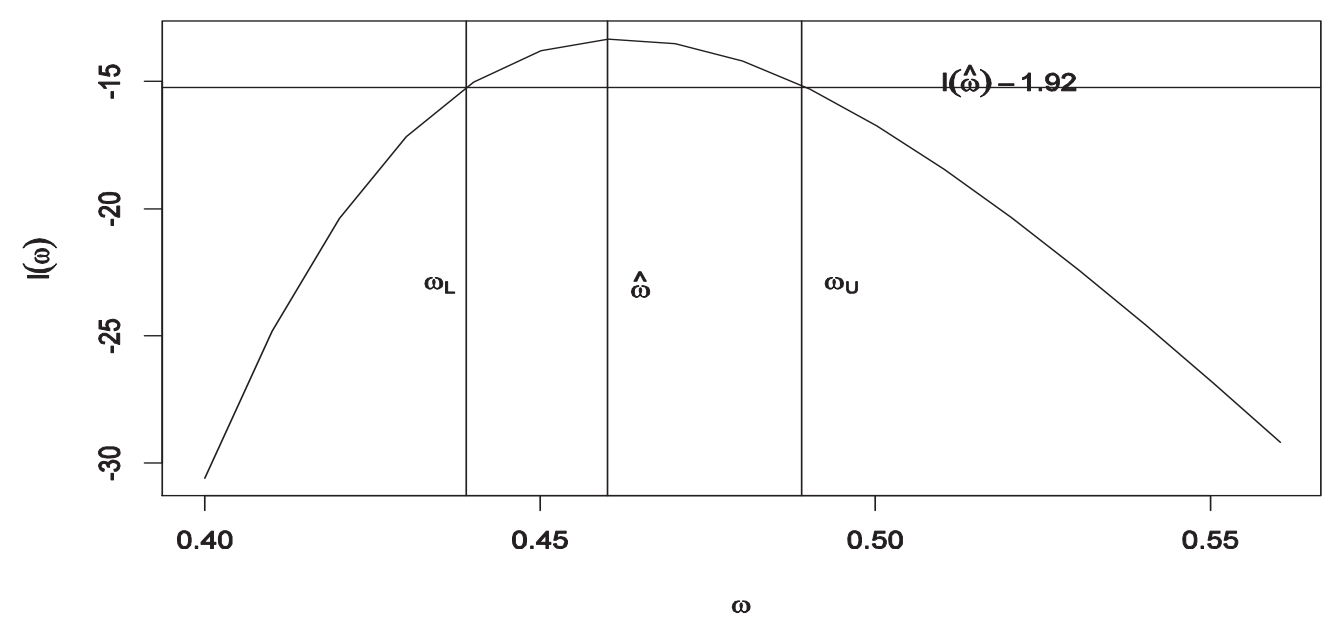

Fig. 8. Profile likelihood function and $95 \% \mathrm{CI}:\left(\omega_{L}, \omega_{U}\right)$ for the crime sociology data.

distribution would not be an appropriate model for these data. This can also be seen by the likelihood ratio test $\Lambda=2(114.95)=229.9$.

\section{Conclusion}

It has been shown that the LMB distribution can be very useful in a variety of applications since it allows for over-dispersion and under-dispersion relative to the standard binomial distribution. The first four non-central and central moments are obtained. Moreover, the LMBD has been used for testing the appropriateness of the standard binomial distribution to the data using the likelihood ratio test and the confidence interval has been obtained for its parameters using the profile likelihood. The simulation procedures for generating data from multiplicative-binomial distribution using R-software are illustrated. It is shown that the LMB distribution could outperform the standard binomial and beta-binomial distributions using four applications to simulated and real data.

\section{Acknowledgment}

The author is greatly grateful to two reviewers for careful reading, valuable comments and highly constructive suggestions that have led to clarity, better presentation and improvements in the manuscript. 


\section{References}

[1] Altham, P. (1978). Two generalizations of the binomial distribution. Applied Statistics, 27, 162-167.

[2] Bohning, D., Dietz, E. and Schlattmann (1997). Zero-inflated count models and their applications in public health and social science. In: Applications of latent trait and latent class models in the social sciences, ed. by Jurgen R. and Langeheine, WAXMANN Verlag.

[3] Banik, S. and Kibria, B.M.G. (2009). On some discrete distributions and their applications with real life data. Journal of Modern Applied Statistical Methods, 8, 423-447.

[4] Bowman, D. and George, E.O. (1995). A saturated model for analyzing exchangeable binary data: application to clinical and development toxicity studies. Journal of American Statistical Association, 90, 871-879.

[5] Cox, D.R. (1972). The analysis of multivariate binary data. Applied Statistics, 21, 113-120.

[6] Habib E.A. (2010). Estimation of log-linear binomial distribution with applications. Journal of Probability and Statistics, 10, 1-13.

[7] Kolev, N. and Paiva, D. (2008). Random sums of exchangeable variables and actuarial applications. Insurance: Mathematics and Economics, 14, 147-153.

[8] Kibria, B.M.G. (2006). Applications of some discrete regression models for count data. Pakistan Journal of Statistics and Operation Research, 2, 1-16.

[9] Kuan, J., Peck, R.C. and Janke, M.K. (1991). Statistical methods for traffic accident research, in: Proceedings of the 1990 Taipei Symposium in Statistics, June 28-30, 1990, ed. by Min-Te Chao and Philip E. Cheng. Taipei, Institute of Statistical Science.

[10] Lambert, D. (1992). Zero-inflated Poisson regression, with application to defects in manufacturing. Technometrics, 34, 1-14.

[11] Lovison, G. (1998). An alternative representation of Altham's multiplicative-binomial distribution. Statistics \& Probability Letters, 36, 415-420.

[12] Pawitan, Y. (2001). In all likelihood: Statistical modeling and inference using likelihood. ( ${ }^{\text {st }}$ Ed.), Clarendon Press, Oxford.

[13] Severini, T.A. (2000). Likelihood methods in statistics. (1 ${ }^{\text {st }}$ Ed.), Oxford University Press.

[14] Siegel, S., and Castellan, N.J. (988). Nonparametric statistics for the behavioral sciences( $2^{\text {nd }}$ Ed.), New York, NY: McGraw-Hill.

[15] Venzon, D.J. and Moolgavkar, H. (1988). A Method for computing profile-likelihood-based confidence intervals. Applied Statistics, 37, 87-94.

[16] Yu, C. and Zelterman, D. (2002). Sums of dependent Bernoulli random variables and disease clustering. Statistics \& Probability Letters, 57, 363-373.

[17] Yu, C. and Zelterman, D. (2008). Sums of exchangeable Bernoulli random variables for family and litter frequency data. Computational Statistics \& Data Analysis, 52, 1636-1649. 